COMMENT. First described by Cogan DG in 1952, congenital ocular motor apraxia (COMA) is a paresis of conjugate purposive gaze, with retained random movements, and compensatory jerking movements of the head. Optokinetic nystagmus is abnormal, with absence of the fast, refixation phase. The patient is unable to follow a fast moving object but can follow a slow moving object horizontally to either side. Jerking of the head redirects the eyes into the desired position. Repetitive blinking helps in correcting fixation. MRI is normal or may reveal agenesis of the corpus callosum, hypoplasia of the cerebellar vermis, and brainstem dysgenesis. In addition to congenital cases, the syndrome may be a sign of brain tumor, Gaucher's disease, ataxia-telangiectasia, and Joubert syndrome. Joubert syndrome and COMA are allelic for the NPHP1 gene for juvenile nephronophthisis (Betz R et al. J Pediatr $2000 ; 136: 828-831)$. The congenital type, originally described as benign, is now known to be associated often with gait ataxia, speech apraxia, behavioral and learning disabilities, and intellectual delay. The present series of COMA cases demonstrates a correlation between delay in walking ability and subsequent gait ataxia, speech problems, and intellectual retardation. Structural abnormalities of the cerebellum and brainstem are demonstrated in more than half the cases.

\title{
NEUROLOGIC ABNORMALITIES IN WILLIAMS SYNDROME
}

The neurologic features of 47 cases of Williams syndrome were determined and a follow-up study was performed on a subgroup of 27 subjects at the Neurorehabilitation Unit, IRCCS Eugenio Medea, Bosisio Parini, Italy. All the patients showed some neurologic deficit, but none was major, and none affected the cranial nerves or peripheral nerves. Proximal hypotonia was found in all but one $(98 \%)$, and mild rigidity in $6(12.8 \%)$. Hyperreflexia was elicited in 20 patients (42.6\%), and Babinski responses in $2(4.3 \%)$. Soft cerebellar signs were frequent: dysmetria (31.9\%), dysdiadochokinesia $(95.7 \%)$, tandem dyspraxia (93.6\%), braking $(59.6 \%)$, and gait ataxia (6.4\%). Extrapyramidal signs were mild and included choreiform movements in $46.8 \%$, dystonia in $42.6 \%$, other involuntary movements in $36.2 \%$, and facial grimacing in $55.3 \%$. Cerebellar signs showed no consistent change whereas extrapyramidal signs, especially dystonia, tended to increase with age from 8 years to $14+$ years, during the 4 -year follow-up $(\mathrm{P}<0.01)$. The extrapyramidal abnormalities are linked to a dysfunction in the nigrostriatal dopaminergic system that increases with the accelerated ageing process, a characteristic of Williams syndrome. (Gagliardi C, Martelli S, Burt MD, Borgatti R. Evolution of neurologic features in Williams syndrome. Pediatr

Neurol May 2007;36:301-306). (Respond: Chiara Gagliardi MD. E-mail: chiara.gagliardi@bp.Inf.it).

COMMENT. Williams syndrome is caused by a deletion in chromosome $7 \mathrm{q} 11.23$ and affects multiple systems, especially cardiac. The patient has an elfin-like facial appearance, infantile hypercalcemia that resolves with age, supravalvular aortic stenosis and hypertension, hyperacusis, and cognitive disorders. Nonverbal functions, visual-motor and spatial perceptions, are weak, whereas expressive language, musical abilities, and facial recognition are relative strengths. The older patient has a "cocktail personality." Less attention has been given to neurological and behavioral symptoms. Some patients with Williams syndrome (WS) have ADHD and have been treated with methylphenidate (Bowden 
HN et al. J Child Neurol 1997;12:248-252). In our ADHD Neurology Clinic, we have seen one patient with WS in the past 10 years. With a structural heart defect, the use of stimulant medication is generally contraindicated. So-called "soft" neurologic signs are a common finding in WS, especially cerebellar and extrapyramidal signs. The present large series of patients with WS, including long-term follow-up observations, documents the changes in neurologic signs with age and the increase in extrapyramidal signs related to an accelerated ageing process.

\section{INFECTIOUS DISORDERS}

\section{DIAGNOSIS AND OUTCOME OF HERPES SIMPLEX ENCEPHALITIS}

Children with herpes simplex encephalitis (HSE) admitted to the Hospital for Sick Children, Toronto, Canada, between Jan 1994 and Dec 2005, were studied prospectively. Sixteen $(5 \%)$ of 322 patients with acute encephalitis fulfilled criteria for HSE (encephalopathy, defined as depressed or altered level of consciousness $>24$ hours, plus additional specific criteria). Median age was 4 years (range, 2 months to 14 years); neonatal cases were excluded. Exposure to HSV was known in $8(50 \%)$. All 16 presented with fever, $11(69 \%)$ with focal seizures, $5(31 \%)$ with hemiparesis, and $2(13 \%)$ with dysphasia. CSF showed pleocytosis $\left(>5 \times 10^{6}\right.$ cells per L) in $15(94 \%), 50$ to $100 \times 10^{6} \mathrm{RBCs}$ per $\mathrm{L}$ in 3 $(19 \%)$, and elevated protein levels in $8(50 \%)$. CSF PCR evidence of HSV was present in 12 (75\%) patients, and 4 with negative CSF PCR had $->>$ fourfold increase in complement fixation titers in convalescent cf acute serum. In 2 patients, PCR was negative on day 1 and positive on day 3 or 7 . HSV-1 was detected in $10(83 \%)$ and HSV-2 in $2(17 \%)$. Other identified pathogens included mycoplasma pneumoniae in 4 patients, and 1 case each with HHV-6, enterovirus, Epstein-Barr virus, influenza A, and parainfluenza 3. EEG showed generalized slowing in $13(81 \%)$, and periodic lateralizing epileptiform discharges (PLEDS) in $2(13 \%)$. CT or MRI abnormalities consistent with HSE (localized edema, mass effect, or hemorrhage) were present in 14 (88\%). Four (25\%) had infarction and hemorrhage. Of 6 cases with negative CTs, 4 had abnormal MRIs. Despite treatment with acyclovir within the first 3 days and for 14 to 21 days, $10(63 \%)$ patients had adverse neurologic outcomes. Sequelae included seizures in $7(44 \%)$, global developmental delay in $4(25 \%)$, and hemiplegia in $2(13 \%)$. No patient died. Outcome was not correlated with age, clinical features, CSF, EEG or neuroimaging abnormalities. (Elbers JM, Bitnun A, Richardson SE et al. A 12-year prospective study of childhood herpes simplex encephalitis: Is there a broader spectrum of disease? Pediatrics Feb 2007;119:e399-e407). (Respond: Ari Bitnun MD MSc FRCPC, Division of Infectious Diseases, Hospital for Sick Children, University of Toronto, 555 University Ave, Toronto, Ontario, MSG 1X8, Canada).

COMMENT. HSV accounts for $5 \%$ of all cases of acute encephalitis in children. The classic clinical presentation of HSE, with fever, altered level of consciousness, focal motor seizures, dysphasia, and hemiparesis, occurs in $75 \%$ of cases. Other presentations include ataxia, decreased visual acuity, tremor, or generalized tonic-clonic seizures. A single negative CSF PCR does not exclude a diagnosis of HSV as the cause of acute encephalitis, In patients with typical clinical presentation and consistent MRI findings, a second lumbar puncture is recommended after 3 days. The absence of CSF pleocytosis, elevated protein, and 